\title{
Histone H3K4me3 modification is a transgenerational epigenetic signal for lipid metabolism in Caenorhabditis elegans
}

Qinghua Zhou ( $\nabla$ gene@email.jnu.edu.cn )

Jinan University https://orcid.org/0000-0003-0468-9266

Qin-Li Wan

Jinan University https://orcid.org/0000-0003-2864-912X

Xiao Meng

Jinan University

Chongyang Wang

Jinan University https://orcid.org/0000-0003-2254-1871

Wenyu Dai

Jinan University https://orcid.org/0000-0003-4669-5787

Zhenhuan Luo

Jinan University https://orcid.org/0000-0003-1375-0882

Xiaodie Fu

Jinan University

Jing Yang

Jinan University https://orcid.org/0000-0002-9498-608X

Qunshan Ye

Jinan University https://orcid.org/0000-0003-1785-2931

Zhan-Hui Zhang

Jinan University

\section{Article}

Keywords: transgenerational epigenetic inheritance, C. elegans, lipid accumulation, histone H3K4me3 modification

Posted Date: December 18th, 2020

DOI: https://doi.org/10.21203/rs.3.rs-111852/v1

License: (c) (1) This work is licensed under a Creative Commons Attribution 4.0 International License. Read Full License 
Version of Record: A version of this preprint was published at Nature Communications on February 9th, 2022. See the published version at https://doi.org/10.1038/s41467-022-28469-4. 


\section{Abstract}

As a major risk factor to human health, obesity presents a massive burden to people and society. Interestingly, the obese status of parents could affect progeny's lipid accumulation through multigenerational epigenetic inheritance. To date, many questions remain as to how lipid accumulation leads to signals that are transmitted across generations. In this study, we established a model of $C$. elegans fed with a high fat diet (HFD) that led to obvious lipid accumulation, which can be propagated their progeny. Using this model, we discovered that transcription factors DAF-16/FOXO and SBP-1, nuclear receptors NHR-49 and NHR-80, and delta- 9 desaturase (fat -5 , fat -6 , and fat 7 ) are required for transgenerational fat accumulation. Additionally, histone H3K4 tri-methylation (H3K4me3) marks genes related to lipid metabolism and increases their transcription response to multigenerational obesogenic effects. In summary, this study establishes that a network of lipid metabolic genes and chromatin modifications work together to achieve multigenerational obesogenic effects.

\section{Introduction}

Metabolic diseases are the result of a combination of genetic and environmental effects. Common environmental factors affecting metabolic diseases include nutrition, microbiome, exercise, and individual behaviors 1,2,3,4. Interestingly, studies in animal models and humans have indicated that environmental influences that parents experience can not only influence their own health, but also affect the health of their descendants, initiating a pass-down of disease risk across generations. For the incidence of obesity and type II diabetes (T2D), besides genetic and environmental factors, family history, especially the parental experiences before and during pregnancy, is also now recognized as an important factor ${ }^{5}$. The study of Netherlands' famine period "hunger winter" was very instructive in understanding how environmental stress stayed as a transgenerational physiological memory. During the sudden and severe famine, children born to pregnant mothers who experienced undernutrition were found to possess a higher risk of metabolic disorders ${ }^{6,7,8}$. In a study of Caenorhabditis elegans (C. elegans), it was also found that information from parents who experienced acute periods of starvation can be passed on to

their progeny $9,10,11$. In modern societies, people no longer live in fear of famine. Instead, we live in a condition of over-nutrition, which is followed by a high incidence of obesity, and thus a variety of metabolic diseases such as type II diabetes, hypertension, and coronary heart disease. Therefore, it is important to study whether and how obesity induces multigenerational inheritance, and the nature of the underlying molecular mechanisms.

The nematode $C$. elegans is a relevant and tractable model for the study of multigenerational obesogenic effects. Nematode genes for lipid metabolism are mostly conserved in mammalian models, with core lipid metabolic pathways in $C$. elegans having homologs in humans. The transgenerational epigenetic inheritance (TEI) machinery that has until now been elucidated is also conserved across species. For example, histone modifications involving methylation of $\mathrm{H} 3 \mathrm{~K} 4, \mathrm{H} 3 \mathrm{~K} 9$, and $\mathrm{H} 3 \mathrm{~K} 27$, and inherited RNAs are required for TEl among different species from $C$. elegans to mice ${ }^{12,13,14,15}$. Recently, some progress has 
been made in the study of TEl using nematodes as a model. For example, $C$. elegans learn to avoid pathogens and transmit the ability to avoid dangerous conditions to their progeny for 4 generations ${ }^{16,17}$. An artificial environment such as Bisphenol (BPA) exposure can cause transgenerational inheritance, with one-generational parental (P0) exposure BPA leading to a decrease of H3K9me3 and H3K27me3 levels

for 5 generations ${ }^{14,18}$. In our recent study, we found that hormetic heat stress-induced survival advantage could be passed down to progeny though histone $\mathrm{H} 3 \mathrm{~K} 9 \mathrm{me} 3$ and DNA $\mathrm{N}^{6}$-mA modifications ${ }^{19}$ (in press).

The question of whether, and if so how, TEl plays a role in lipid metabolism has attracted the attention of researchers in recent years. For example, it was reported that the TEl obesogenic effects of sulfomethoxazole are associated with histone H3K4me3 modification ${ }^{20}$. However, some mechanisms of the underlying multigenerational obesogenic effects remain unclear. For example, how are genes related to fat metabolism linked to chromatin modifiers to achieve the lipid metabolism-associated TEI? In this study, we used a high-fat diet (HFD, egg yolk) to feed $C$. elegans to establish a lipid accumulation model. Using this model, we characterized the critical factors for implementing of regulation of lipid metabolism and transmitting transgenerational memories in descendants from parents fed with HFD.

\section{Results}

\section{Lipid accumulation from the HFD exposure can be transmitted across generations}

Supplementation of different lipids, including oleic acid (OA) or palmitoleic acid (PA), in food, can significantly increase fat accumulation in $C$. elegans ${ }^{21}{ }^{22}$. Similarly, using Oil-Red-O (ORO) staining, we found that the ORO level was obviously elevated when animals were fed with OA, PA, cholesterol, or egg yolk compared with the OP50 medium, and that supplementation with egg yolk showed the most significant lipid accumulation (Fig 1a). Therefore, in the subsequent assays, we used egg yolk as a highfat diet (HFD) to feed $C$. elegans and establish a fat accumulation model. To test whether the HFDinduced lipid accumulation phenomenon can transmit to the descendants raised on OP50, we randomly selected one wild-type hermaphrodite worm and obtained a population with the same genetic background through its self-fertilization. Then we fed wild-type worms with the HFD and bleached the mothers to obtain F1, F2, and Fn generations (Fig 1b). We found that the ORO levels were significantly increased, in either the HFD-fed parents or their F1 and F2 progeny (fed with normal OP50) (Fig 1c and d), but did not increase ORO levels of their F3 descendants (data not shown), suggesting that the HFD can induce a multigenerational epigenetic inheritance phenotype on descendants from a single exposure of the P0 generation. Considering that the F1 generation may have been exposed to the HFD while still inside the mother, in which case the obesogenic effect in the F1 worms would be considered intergenerational inheritance, not trans-generational inheritance. Therefore, we fed PO generation animals with HFD from L1 to L4 larvae, then transferred them to NGM plates with normal food to prevent F2 primordial germ cells (PGCs) from exposure. We found that pre-exposure of P0 worms to HFD until L4 larvae could also induce 
the lipid accumulation of their naive F1 and F2 progeny (Fig 1e), suggesting that HFD can induce a transgenerational epigenetic inheritance (TEI) obesity phenotype.

In our heritable obesity effect model, we found that F2 populations exhibited a minor lipid accumulation phenotype. One concern is that HFD-induced fat accumulation could trigger a maternally heritable response in a dose-dependent manner instead of TEl effect. In order to eliminate this concern, we exposed multiple consecutive generations of animals to HFD. We found that, the exposure of four consecutive generations of animals (P0, F1, F2, and F3), three (F1, F2, and F3), two (F2, and F3), or one (F3), did not affect lipid accumulation their recovered F4 or F5 descendants (Fig $1 \mathrm{f}$ and g). In addition, we observed that lipid accumulation induced by oleic acid (OA) or palmitoleic acid (PA) can also transmit to their naive progeny (Fig S1C-e). Altogether, these results demonstrated that exposure of HFD induces transgenerational obesity effect.

To further elucidate the TEl phenotype and possible mechanisms induced by the HFD, we performed RNA sequencing (RNA-seq) analysis. Consistent with a previous study, functional annotation analysis indicated that genes regulated after feeding with the HFD were enriched in the innate immune response, metabolism and lipid metabolism (Fig S1a). Notably, a series of genes which were regulated by the HFD in the P0 generation overlapped with those regulated in the F1 and F2 naive progeny derived from P0 parents given the sole exposure to the HFD (Fig S1b). Collectively, these results demonstrated that the HFD can induce a memory of fat accumulation that can be transmitted to descendants.

\section{HFD-induced transgenerational inheritance is mediated by nuclear receptors NHR-49, NHR-80, and transcription factors SBP-1 and DAF-16}

To determine the molecular mechanisms underlying the HFD-induced TEl, we evaluated the contribution of several nuclear receptors and transcription factors which play essential roles in lipid metabolism in $C$. elegans. SBP-1, a sterol regulatory element-binding protein (SREBP), is a crucial transcription factor governing fat metabolism ${ }^{21}$. NHR-49, a functional homolog of mammalian peroxisome proliferator activated receptor (PPAR) alpha, and NHR-80, a homolog of mammalian hepatocyte nuclear factor 4 (HNF4), are both important nuclear hormone receptors involved in the control of fat consumption and fatty acid composition in $C$. elegans $23,24,25,26$. Forkhead transcriptional factor DAF-16/FOXO, a central downstream effector of the insulin/insulin-like growth factor (IGF) signaling pathway, is a critical and well-conserved metabolic regulator 27,28 . Our results demonstrated that the elevated fat level induced by HFD in P0 was not affected in a sbp-1 loss-of-function (lof) mutant (Fig 2a), an $n h r-80$ lof mutant (Fig 2b) or an $n h r-49$ lof mutant (Fig 2c). However, lipid accumulation was abrogated in F1 or F2 descendants of these mutants. By contrast, the elevated lipid phenotype induced by HFD was abolished in P0 parents as well as in their recovery F1 and F2 progeny in the daf-16 lof mutant (Fig 2d). These results indicated that SBP-1, NHR-49 and NHR-80 work in the F1 generation to mediate TEl of fat accumulation induced by the 
HFD; whereas DAF-16 not only act in the F1 generation, but also is required for the execution of the lipid metabolism response in the $\mathrm{P} 0$ generation.

Here, considering that daf-16 contributed to regulation of lipid metabolism in P0 generation, to more definitively identify the role for daf-16 in F1 generation, we used RNAi to knock down daf-16 exclusively in the F1 generation derived from the P0 parents fed the HFD. We found that specific knockdown of daf-16 in the F1 generation also abrogates the elevated fat level (Fig 2e \& f). Moreover, we also performed similar analyses using $n h r-49, n h r-80$ and $s b p-1$ RNAi. We found that silencing $n h r-49, n h r-80$ and $s b p-1$ in F1 progeny also abolished their lipid accumulation (Fig S2a-e), suggesting that daf-16, nhr-49, nhr-80 and $s b p-1$ act in the progeny to increase lipid level.

DAF-16 responses to environmental stress by activating a series of target genes ${ }^{28}$. We found that the mRNA level of the target genes of DAF-16 (sod-3 and dod-3) was significantly upregulated in P0 fed with the HFD or their naive F1 progeny (Fig S3c). The expression of SOD-3::GFP was consistent with the mRNA level of sod-3 (Fig S3a and b). Altogether, these results indicated that DAF-16 functioned in response to altered lipid metabolism induced by HFD in PO and their recovered progeny.

To more definitively dissect whether the role of daf- $16, n h r-49, n h r-80$ and $s b p-1$ is to implement regulation of lipid level (an "executor"), to transmit the heritable memories (a "transmitter"), or both, we used RNAi to silence daf-16, nhr-49, nhr-80 and sbp-1 exclusively in the P0 generation, and then analyzed the fat level of $\mathrm{F} 1$ generation raised on OP50 (Fig $2 \mathrm{~g}$ ). As reported in our recent study ${ }^{19}$ (in press), if a gene is a transmitter, the silencing of that gene in the P0 generation will prevent the transmission of transgenerational memory, which will result in the elimination of the lipid accumulation of the F1 generation. Alternatively, if a gene only functions as an executor, the silencing of that gene in the $\mathrm{P} 0$ generation should only influence the lipid level of PO generation, so that we would still detect the elevation of fat level in F1 generation. The results showed that silencing of $n h r-49$ and $n h r-80$ did not abrogate the lipid accumulation of $\mathrm{F} 1$ progeny (Fig $2 \mathrm{i}$ and j), suggesting that $n h r-49$ and $n h r-80$ are purely executors but not transmitters; the silencing of $d a f-16$ or $s b p-1$ in P0 generation caused the loss of lipid accumulation of F1 (Fig $2 \mathrm{~h}$ and $\mathrm{k}$ ), indicating their roles as transmitters.

Taken together, our results demonstrated that daf-16, nhr-49, nhr-80 and $s b p-1$ were required for HFDinduced TEl of lipid accumulation. Among them, $n h r-49$ and $n h r-80$ functioned solely as executors; sbp- 1 was responsible for transmitting the heritable memories, though we could not rule out its role as an executor; for $d a f-16$, it not only functions as an executor to regulate lipid accumulation, but also as a transmitter to pass down heritable memory to progeny.

\section{Delta-9 desaturase mediated the heritable memories}

In $C$. elegans, delta-9 desaturases are well-characterized targets downstream of highly conserved transcriptional factors SBP-1, NHR-49, NHR-80 and DAF-16 (Fig 3a) 11, 25, 29, 30. Because SBP-1, NHR-49, 
NHR-80 and DAF-16 have been detected to mediate TEl of lipid accumulation in our settings, we wondered whether delta-9 desaturases participated in the TEl of lipid accumulation. We used the delta-9 desaturases-related mutants including fat -5 , fat -6 , and fat -7 lof mutants, and fat -5 ; fat -6 and fat -5 ; fat -7 double mutants to test our hypothesis. Our results showed that the fat elevated phenotype remained largely unaffected in the P0 generation of these mutants; however, these mutations abrogated the elevated fat level in F1 progeny (Fig 3b-f). These results revealed that delta-9 desaturases function in TEI induced by the HFD. To more definitively specify the role of delta-9 desaturases in HFD-induced TEl, we chose fat- 5 as the representative of desaturase to conduct two sets of RNAi experiments similar to that performed in the above-mentioned treatments (daf-16, nhr-49, nhr-80 and sbp-1). We found that silencing fat-5 in $\mathrm{F} 1$ abolished the lipid accumulation (Fig 3g), however, silencing fat-5 in P0 generation did not affect the phenotype of fat accumulation in their recovered F1 progeny (Fig 3h), suggesting that delta-9 desaturases just execute regulation of lipid metabolism in HFD-induced TEl, but not transmit heritable memory.

\section{Memory of HFD-induced lipid metabolism was conferred by histone H3K4me3 modification}

HFD-induced lipid accumulation in parents could just pass down to F2 generation, and F3 is back to normal lipid level, suggesting that HFD might induce epigenetic changes rather than genetic mutations in descendants. Reportedly, a set of chromatin-modifying enzymes, including deacetylases and H3K4me3 methyltransferase, influence lipid metabolism ${ }^{31,32}$. To understand whether methyltransferase mediated the HFD-induced TEl of obesogenic effect, we performed a targeted mutant screen by selecting genes which participated histone methylation (Fig S4a and b). We found that loss of wdr-5.1, a H3K4me3 complex component, abolished the lipid accumulation in progeny (Fig 4a). Furthermore, wdr-5.1 did not execute in responding to lipid metabolism induced by the HFD, because ORO signaling of the wdr-5.1 mutant remained mostly increased in the P0 parents (Fig 4a). Moreover, we detected that the mRNA level of $w d r-5.1$ was slightly (but statistically significant) upregulated when animals were fed with the HFD compared with the control (Fig S4c). By contrast, our results showed that other histone modifications including H3K27me3 and H3K36me3 did not contribute to the TEI induced by the HFD, because lipid accumulation induced by the HFD was not abrogated in the P0, F1 and F2 generations in the loss of function H3K27 demethylase mutant jmjd-3. $1^{33}$ and in the loss of function H3K36 methyltransferase mutant met- $^{14}$ (Fig S4a and b). Furthermore, we observed that H3K4me3 methylation was significantly increased in the P0 generation fed with HFD (Fig 4b) and in their recovered progeny (Fig 4b, c and d). Moreover, upregulation of H3K4me3 methylation induced by feeding with HFD was abolished in a wdr-5.1 mutant (Fig S4d).

To more definitively characterize the role of $w d r-5.1$ in HFD-induced TEl, we also conducted two sets of RNAi experiments similar to that performed in the above-mentioned treatments (daf-16, nhr-49, nhr-80 and $s b p-1$ ). We found that silencing $w d r-5.1$ in F1 abrogated the lipid accumulation (Fig S4e), and silencing $w d r-5.1$ in P0 generation also abolished the phenotype of elevation of fat level in their recovered 
F1 progeny (Fig S4f), suggesting the role of $w d r-5.1$ as a transmitter instead of an executor. Collectively, these results suggested that histone H3K4me3 modification plays a specific role in transmitting the heritable memory induced by the HFD.

To test whether lipid accumulation induced by the HFD is associated with heritable changes of H3K4me3 at specific loci, we used CHIP-qPCR and found that H3K4me3 occupancy was significantly increased at the promoter of several genes related to lipid metabolism (Fig 4f). These genes are also significantly upregulated, as shown by the RNA-seq analysis of the P0, F1 and F2 generations. In addition, the mRNA level of these genes was confirmed to be elevated by RT-qPCR (Fig $4 \mathrm{~g}$ ).

We further investigated whether the transcription factors ( $s b p-1$ and daf-16) and nuclear receptors ( $n h r-49$ and $n h r-80$ ), which have been identified to mediate HFD-induced TEl, also regulated the levels of histone H3K4me3 modification. We found that all mutations (daf-16, nhr-49 and nhr-80) except sbp-1 had no effect on H3K4me3 modification in animals fed with the HFD when compared with controls (Fig 4e, S5b, S5c and S5d). In addition, the elevated transcription level of $w d r-5.1$ induced by the HFD was abrogated in the sbp-1 mutant (Fig S4C). These findings suggested that $s b p-1$ might mediate the HFD-induced memory transmission of lipid accumulation through upregulating $w d r-5.1$ expression to increase the H3K4me3 level.

\section{Germ-to-soma communication in the HFD-induced transgenerational inheritance}

To detect tissues in which $d a f-16$ and sbp-1 function in response to lipid metabolism and in regulating histone modifications, respectively, we conducted tissue-specific gene knockdown assays by using strains which can process RNAi efficiently only in specific tissues, such as germline, muscle, or neuron (Fig 5a). Firstly, we performed the tissue-specific RNAi of daf-16 to detect the ORO staining. We found that muscle-specific RNAi of daf-16 in the PO generation led to the suppression of the lipid accumulation (Fig 5g). However, other tissue-specific RNAi, including in the intestine, neuron or germline, could not compromise the elevated fat level (Fig 5h-k).

We then performed tissue-specific knockdown of $s b p-1$ to assess the variation of histone H3K4me3 induced by the HFD. We found that intestine-specific, muscle-specific, germline-specific, or intestine- and germline-specific RNAi of $s b p-1$ abolishes the elevated H3K4me3 modification levels (Fig $5 b$-f). These results indicated that the function of $s b p-1$ to regulate the variation of H3K4me3 modification induced by the HFD in several tissues may be redundant. Collectively, tissue-specific RNAi studies demonstrated that among tissues, communication across generations may coordinately regulate TEl of lipid accumulation induced by the HFD.

\section{Discussion}


During the last few decades, the number of obese people has been growing rapidly in many countries, causing a huge economic burden to society ${ }^{34}$. However, for metabolic diseases such as obesity and T2D, besides genetic and environmental factors, family history has increasingly been considered to be an important factor. In fact, genetic background only contributes $~ 5 \%-10 \%$ of overall risk for developing T2D 5 . Recently, considerable efforts have been done to elucidate the TEl-associated obesity induced by overnutrition. However, many mechanisms remain uncovered. In this work, we use $C$. elegans as a model, and induce lipid accumulation by feeding HFD (egg yolk) to $C$. elegans. Our results demonstrated that an obesity effect in parents in $C$. elegans can be induced by feeding egg yolk, and could be transmitted to their naive progeny which have never been exposed to the HFD.

In addition, our study revealed that TEl of lipid accumulation was mediated by histone H3K4me3 modification. Moreover, we found that among factors identified in TEl of fat accumulation, tissue-tissue communication exists to execute the regulation of TEl effect. Consistent with previous reports ${ }^{28,29}$, we found that DAF-16 is an essential regulator in the response to variation of lipid metabolism induced by the HFD. Furthermore, we observed that $s b p-1, d a f-16, n h r-49, n h r-80$, and delta- 9 fatty acid desaturase genes (fat-5, fat-6, and fat-7) were responsible for TEl of obesogenic effects. Among them, $n h r-49$ and nhr-80 contributed to regulating lipid metabolism; sbp- 1 transmitted transgenerational memory; while daf16 acted in both regulation of lipid metabolism and transmission of obesogenic information. Strikingly, $s b p-1$ itself also contributed to regulating the level of histone H3K4me4 modification. Consistent with our observations, a previous study has discovered that in $C$. elegans, a complex transcriptional network consisting of SBP-1, MDT-15, DAF-16, NHR-49, and NHR-80 regulates the activity of the delta-9 fatty acid desaturase genes (fat -5 , fat- 6 , and fat- 7 ). In turn, these factors affect the modification of H3K4me3, ultimately affecting the lifespan and lipid metabolism of the nematodes ${ }^{32}$. Taken together, these results suggest that, upon animals being fed with the HFD, the stress of lipid accumulation in the parental generation induces activity of lipid metabolic transcription factors sbp-1, daf-16, nhr-49, and nhr-80. At the same time, $s b p-1$ regulates histone $\mathrm{H} 3 \mathrm{~K} 4 \mathrm{me} 3$ modification, and establishes the epigenetic marks in descendants. In turn, the H3K4me3 marks in the progeny promote the recruitment of lipid metabolismrelated genes (i.e. $s b p-1$ and daf-16) and facilitate the activation of the response to lipid metabolism and ultimately reset the metabolic processes, thereby completing the TEl of obesity effect (Fig 6).

In this study, we used egg yolk as the HFD to induce the obesity model of $C$. elegans. We found that, except for daf-16, loss of function of other genes, including $n h r-49$, nhr-80, and $s b p-1$ which are recognized as central regulators of lipid metabolism, cannot completely block lipid accumulation induced by the HFD in the PO generation. A possible explanation is that the composition of the egg yolk is complex, so that these genes only partially mediate the egg yolk-induced lipid accumulation. Because our work focused on the transgenerational inheritance of lipid accumulation, we have not yet fully elucidated the underlying details of molecular mechanisms which regulate lipid metabolism changes induced by egg yolk. This will be a subject of future work.

In addition, it is still unclear how $s b p-1$ regulates the level of histone H3K4me3 modification in this work. Epigenetic mechanisms include chromatin structure and modification, DNA methylation and noncoding 
RNAs. In this study, we identified histone H3K4me3 modification as contributing to the TEl of obesity effect, however, the function, if any, of DNA methylation and noncoding RNAs remains ambiguous. Therefore, more work is needed to further disentangle the molecular mechanisms of TEl related to obesity, including how and when the epigenetic memory is established, as well as how lipid metabolism genes interplay with genes involved in epigenetic inheritance. Our research provides evidence that the lifestyle of parents is very important to the metabolic status of offspring in nematodes, and it is tempting to speculate that these findings may be relevant to mammalian systems. Moreover, our study also uncovered an intrinsic molecular mechanism underlying a multigenerational obesity effect.

\section{Materials And Methods}

\section{Nematode strains and maintenance}

C. elegans strains used in this work were obtained from Caenorhabditis Genetic Center (CGC) (University of Minnesota, USA), which is supported by the NIH NCRR. All strains were maintained on standard nematode growth medium (NGM) plates with Escherichia coli (E. coli) OP50 as previously described ${ }^{35}$. All experiments were performed at $20^{\circ} \mathrm{C}$. Strains used in this work were: wild type (N2), RB1304 wdr5.1(ok1417) III., ZR2 jmjd-3.1 (gk384) X., VC1666 met-1(ok2172) I., BX165 nhr-80(tm1011) III., BX107 fat5(tm420) V., BX110 fat-6(tm331) IV; fat-5(tm420) V., BX106 fat-6(tm331) IV., BX160 fat-7(wa36) fat5(tm420) V., BX153 fat-7(wa36) V., CE541 sbp-1(ep79) III., VC870 nhr-49(gk405) I., CF1038 daf-16(mu86) I, CF1553 muls84 [(pAD76) sod-3p::GFP + rol-6(su1006)], NR350 kzls20 [h/h-1p::rde-1 + sur-5p::NLS::GFP], AMJ345 jamSi2 [mex-5p::rde-1(+)] II, TU3401 uls69 [pCFJ90 (myo-2p::mCherry) + unc-119p:::sid-1], MAH23 rrf-1(pk1417) I, VP303 kbls7 [nhx-2p:::rde-1 + rol-6(su1006)], STE70 nhr-80(tm1011) III.

\section{RNA interference}

RNAi was essentially conducted as previously described ${ }^{36}$. The $E$. coli HT115 transformed with vectors expressing corresponding dsRNA and empty vector were derived from the Ahringer library (Source Bioscience, Nottingham, UK). All vectors were confirmed by sequencing. RNAi bacteria were cultured at 37 ${ }^{\circ} \mathrm{C}$ in LB with $100 \mu \mathrm{g} \mathrm{mL}^{-1}$ ampicillin. Freshly prepared bacteria were spotted on NGM plates with $1 \mathrm{mM}$ isopropyl-B-D-thiogalactoside (IPTG) before using. All RNAi treatments began from synchronized L1 larvae.

\section{Fatty acid supplementation}

All fatty acids and cholesterol used in this study were purchased from Sigma-Aldrich (Munich, Germany). The fatty acid supplementation protocol was performed as previously described ${ }^{32}$. A final concentration of $4 \mathrm{mM}$ oleic acid (OA), $4 \mathrm{mM}$ palmitic acid (PA) and $50 \mathrm{mg} / \mathrm{mL}$ cholesterol were used for experiments. 
For egg yolk supplementation, the cooked egg yolk is ground and mixed with OP50 (volume ratio, 1:5) to provide the $C$. elegans a food supplement. The mixture of egg yolk and bacteria is freshly made before each experiment.

\section{Conditions for inducing lipid accumulation}

To induce lipid accumulation, 500 synchronized L1 larvae were raised on NGM plates with or without the HFD. To obtain F1 generation worms, gravid day 1 adults treated with or without the HFD were bleached and hatched overnight in $10 \mathrm{~mL}$ M9 buffer, and subsequently grown on NGM plates without the HFD. This process was repeated to obtain the Fn generations. For worms fed with HFD until L4 larvae, synchronized L1 larvae were grown on NGM plates with HFD until L4 larvea, then transferred them to NGM plates with normal food, Fn generations animals were obtained using similar protocol above mentioned.

\section{Oil Red 0 staining and quantification}

Oil Red O (ORO) staining and quantification were conducted as previously described ${ }^{36}$. Briefly, all strains were cultured as described under conditions to accumulate lipid to obtain P0, F1 and F2 generation animals. Then animals were fixed, ORO stained, and mounted to agar plates for imaging at 200x magnification with a Nikon Ti2-U microscope. The same exposure setting were used across all condition within each experiment. The ORO intensity (arbitrary unit, a.u.) was measured using ImageJ processing software as previously described after background removed and greyscale converted ${ }^{37}$. Statistical significance was analyzed using two-tailed Student's t test by GraphPad Prism. At least 30 worms were used per experiment, and the experiment was repeated at least three times.

\section{Quantitative RT-PCR}

RNA extraction and real-time qPCR were conducted as previously described ${ }^{36}$. Briefly, about 4000 worms were used to extract total RNA with RNAiso Plus (Takara). Then RNA was reverse transcribed into cDNA using a cDNA Reverse Transcription Kit (ABclonal) as per the manufacturer's instructions. Real-time qPCR was conducted in triplicate for each gene using a CFX96 Real-Time PCR system (Biorad) with SYBR Green select master mix (ABclonal). The quantification was computed by $2^{-\Delta \Delta C t}$ method after being normalized to gene $c d c-42$. The primers are summarized in the Table S1, Supporting information.

\section{RNA-seq}

Page $11 / 24$ 
Total RNA was extracted using RNAiso Plus (Takara). The RNA was then sequenced by the WuXi App Tec RNA-seq service $(n=3)$. Genes with a greater than twofold change and False Discovery Rate $(F D R)<0.01$ were defined as differentially expressed genes (DEG). Functional annotation of DEG was analyzed through various databases, including Gene Ontology (GO), Kyoto Encyclopedia of Gene and Genomes (KEGG) and EuKaryotic Ortologous Groups (KOG). HFD-induced DEG were assigned functional categories using the Database for Annotation, Visualization and Integrated Discovery (DAVID).

\section{CHIP-qPCR}

Chromatin immunoprecipitation (CHIP) experiments were performed on embryos as previously described 19 (in press). Embryos were collected and flash-frozen in liquid nitrogen. Embryo pellets (50-100 $\mu \mathrm{L}$ ) were incubated with CHIP cross-linking buffer (M9 containing $2 \%$ formaldehyde (Sigma)) at room temperature for $30 \mathrm{~min}$. After quenching with glycine ( $125 \mathrm{mM}$ final), the pellets were washed three times in PBS. Afterwards, samples were resuspended in FA buffer (50 mM HEPES/KOH [pH 7.5], $1 \mathrm{mM}$ EDTA, $1 \%$ Triton $\mathrm{X}-100,0.1 \%$ sodium deoxycholate, and $150 \mathrm{mM} \mathrm{NaCl}, 1 \%$ proteinase inhibitor cocktail (Roche)) and sonicated with a Bioruptor at maximum power for $30 \mathrm{~s}$ on and $30 \mathrm{~s}$ off. Samples were then centrifuged at $12,000 \mathrm{rpm}$ for $10 \mathrm{~min}$ at $4{ }^{\circ} \mathrm{C}$, and the supernatants were stored at $-80^{\circ} \mathrm{C}$. For H3K4me3 CHIP experiments, chromatin extract (1 mg of total protein) was thawed, precleared, then immunoprecipitated with $5 \mu \mathrm{L} \mathrm{H} 3 \mathrm{~K} 4 \mathrm{me} 3$ antibody (Millipore $04-745$ ) at $4{ }^{\circ} \mathrm{C}$ for $12-16 \mathrm{~h}$. Next, $40 \mu \mathrm{L}$ pre-blocked SureBeads ${ }^{\mathrm{TM}}$ Starter Kit Protein G (Bio-Rad) were added, and the tubes rotated at $4{ }^{\circ} \mathrm{C}$ for $4 \mathrm{~h}$. Beads were washed with $1 \mathrm{~mL}$ of the following buffers for $5 \mathrm{~min}$ at room temperature: two times FA buffer, once with FA- $1 \mathrm{M} \mathrm{NaCl}$ buffer (50 mM HEPES/KOH [pH 7.5], 1 mM EDTA, 1\% Triton X-100, 0.1\% sodium deoxycholate, and $1 \mathrm{M}$ $\mathrm{NaCl}$ ), once with FA-500 mM NaCl buffer (50 mM HEPES/KOH [pH 7.5], $1 \mathrm{mM}$ EDTA, 1\% Triton X-100, $0.1 \%$ sodium deoxycholate, and $500 \mathrm{mM} \mathrm{NaCl})$, twice with TEL buffer $(0.25 \mathrm{M} \mathrm{LiCl}, 1 \% \mathrm{NP}-40,1 \%$ sodium deoxycholate, $1 \mathrm{mM}$ EDTA, $10 \mathrm{mM}$ Tris- $\mathrm{HCl}$ (PH 8.0)), and finally with twice $1 \times$ TE buffer. Samples were eluted using elution buffer ( $1 \% \mathrm{SDS}$ in TE with $250 \mathrm{mM} \mathrm{NaCl})$. Supernatants and input samples were incubated with proteinase $\mathrm{K}(0.1 \mathrm{\mu g} / \mathrm{mL})$ for 2 hours at $50^{\circ} \mathrm{C}$ and then de-crosslinked overnight at $65^{\circ} \mathrm{C}$. DNA was purified before preforming qPCR with SYBR. The quantification was computed by the $2^{-\Delta \Delta C t}$ method. The primer from an intergenic region on chromosome IV was used as an internal control, as previously described ${ }^{38}$. Sequences of qPCR primers are listed in Table S2, Supporting information.

\section{Western blot analysis}

Young adult worms treated as described under conditions to accumulate lipid were collected with M9 buffer, washed 3 times and pellets were snap frozen in liquid nitrogen and stored at $-80^{\circ} \mathrm{C}$. Pellets were lysed in RIPA buffer. Next, pellets were ground twice using a TissueLyser at $75 \mathrm{~Hz}$ for 6 min at $4{ }^{\circ} \mathrm{C}$, and centrifuged at $10000 \mathrm{~g}$ at $4^{\circ} \mathrm{C}$. Supernatants were collected. All supernatants were quantified with a BCA Protein Assay Kit. Worm RIPA samples were boiled at $95{ }^{\circ} \mathrm{C}$ for $5 \mathrm{~min}$ before being resolved on SDS-PAGE 
(13.5\%) and transferred to nitrocellulose membrane. The membranes were blocked in $5 \%$ milk, then incubated with primary antibodies to H3K4me3 (1:3000, Millipore $04-745)$ or H3 antibody (1:10000, CST, $\mathrm{H}$ 9715). The primary antibody was visualized using horseradish peroxidase-conjugated anti-rabbit secondary antibody (1:5000) and ECL Western Blotting Substrate.

\section{Worm eggs immunocytochemistry}

For immunostaining of worm eggs, eggs were bleached from gravid worms and washed 3 times with $\mathrm{M} 9$ buffer. Eggs were resuspended in fixing solution $(160 \mathrm{mM} \mathrm{KCl}, 40 \mathrm{mM} \mathrm{NaCl}, 20 \mathrm{mM} \mathrm{Na} 2 \mathrm{EGTA}, 10 \mathrm{mM}$ spermidine $\mathrm{HCl}, 30 \mathrm{mM} \mathrm{NaPIPES}, 50 \%$ methanol, $2 \%$ beta-mercaptoethanol, $2.5 \%$ polyformaldehyde) and frozen in liquid nitrogen for $10 \mathrm{~min}$. Fixed eggs were kept at $-80^{\circ} \mathrm{C}$ for long term storage. Before staining, eggs were thawed and fixed at $4{ }^{\circ} \mathrm{C}$ for $30 \mathrm{~min}$, and washed two times using Tris-Triton buffer $(100 \mathrm{mM}$ Tris-HCl pH 7.4, 1 mM EDTA, 1\% Triton X-100) for 5 min. Eggs were then blocked with PBST-A buffer (PBS PH 7.4, 1\% BSA, 0.5\% Triton X-100, 5 mM sodium azide, 1 mM EDTA) for 20 min and incubated overnight with primary antibodies to H3K4me3 (1:100 in PBST, Millipore 04-745). Eggs then were washed three times, each time for 10 min, with PBST-B (PBS PH 7.4, 0.1\% BSA, 0.5\% Triton X-100, 5 mM sodium azide, $1 \mathrm{mM}$ EDTA), and then incubated with Alexa Fluoro 546 secondary antibody (1:300). DAPI (2 mg mL $\left.{ }^{-1}\right)$ was added to visualize nuclei. Eggs were mounted to a microscope slide and visualized using a Zeiss Axio Imager Z2 with Apotome. 2 microscope at 630 -fold magnification. The fluorescence intensity was analyzed using ImageJ software. Statistical significance was analyzed using two-tailed Student's t test. At least 20 eggs and 10-20 nuclei per egg were used per experiment, and experiments were repeated at least three times.

\section{Fluorescence microscopic imaging}

Quantification of SOD-3 was performed as previously described ${ }^{36}{ }^{19}$ (in press). Briefly, synchronized L1 larvae of CF1553 (SOD-3p::GFP) were treated as described above to induce accumulation of lipid. Young adult worms were mounted to agar plates for imaging using a Nikon Ti2-U fluorescence microscope with $20 \times$ air objectives. To exhibit the fluorescence intensity, 6-7 randomly selected worms were put together to take images. For quantification purpose, each worm was imaged individually. The GFP fluorescence intensity was quantified by Mean fluorescence intensity (Total Intensity/Area) using ImageJ software. At least 30 animals were used per experiment. Statistical analyses were performed using a two-tailed Student's t test.

\section{Declarations}

\section{Conflict of interest}


No conflicts of interests exist in the submission of this manuscript, and the manuscript is approved by all authors for publication.

\section{Author contributions}

Qinghua Zhou and Qin-Li Wan designed the study. Qin-Li Wan, Xiao Meng, Chongyang Wang, Wenyu Dai, Zhenhuan Luo, Jing Yang, Xiaodie Fu and Qunshan Ye conducted the experiments; Qinghua Zhou, Qin-Li Wan and Xiao Meng analyzed the data; Qin-Li Wan wrote the manuscript; Qinghua Zhou and Zhan-Hui Zhang reviewed and edited manuscript; all authors commented on the manuscript.

\section{Acknowledgments}

We would like to thank the Caenorhabditis Genetic Center (CGC) for providing the worm strains, which is funded by the NIH Office of Research Infrastructure Programs (P400D010440). We would like to thank Dr. Xuhui Lai for technical help on our qPCR and RNA-seq procedures. This work was supported by the National Natural Science Foundation (NSFC) of China (Nos. 81670813 and 82001465), National Key R\&D Program of China (2018YFC2002000), the Program of Introducing Talents of Discipline to Universities (111 Project, No. B16021), Natural Science Foundation of Guangdong Province, China (2018A0303131003) and Science and Technology Plan Project of Guangzhou, China (202002030021).

\section{Data and materials availability}

All data needed to evaluate the conclusions in the paper are present in the paper and/or the Supplementary Materials.

\section{References}

1. Miska EA, Ferguson-Smith AC. Transgenerational inheritance: models and mechanisms of non-DNA sequence-based inheritance. Science 354, 59-63 (2016).

2. Song SJ, et al. Cohabiting family members share microbiota with one another and with their dogs. eLife 2, e00458 (2013).

3. Baccarelli A, Bollati V. Epigenetics and environmental chemicals. Current opinion in pediatrics 21, 243-251 (2009).

4. Barres R, Zierath JR. The role of diet and exercise in the transgenerational epigenetic landscape of T2DM. Nature reviews Endocrinology 12, 441-451 (2016). 
5. Voight BF, et al. Twelve type 2 diabetes susceptibility loci identified through large-scale association analysis. Nature genetics 42, 579-589 (2010).

6. Ahmed F. Epigenetics: Tales of adversity. Nature 468, S20 (2010).

7. Roseboom T, de Rooij S, Painter R. The Dutch famine and its long-term consequences for adult health. Early human development 82, 485-491 (2006).

8. Heijmans BT, et al. Persistent epigenetic differences associated with prenatal exposure to famine in humans. Proceedings of the National Academy of Sciences of the United States of America 105, 17046-17049 (2008).

9. Rechavi O, et al. Starvation-induced transgenerational inheritance of small RNAs in C. elegans. Cell 158, 277-287 (2014).

10. Minkina O, Hunter CP. Intergenerational Transmission of Gene Regulatory Information in Caenorhabditis elegans. Trends in genetics : TIG 34, 54-64 (2018).

11. Demoinet E, Li S, Roy R. AMPK blocks starvation-inducible transgenerational defects in Caenorhabditis elegans. Proceedings of the National Academy of Sciences of the United States of America 114, E2689-E2698 (2017).

12. Perez MF, Lehner B. Intergenerational and transgenerational epigenetic inheritance in animals. Nature cell biology 21, 143-151 (2019).

13. Siklenka $\mathrm{K}$, et al. Disruption of histone methylation in developing sperm impairs offspring health transgenerationally. Science 350, aab2006 (2015).

14. Camacho J, et al. The Memory of Environmental Chemical Exposure in C. elegans Is Dependent on the Jumonji Demethylases jmjd-2 and jmjd-3/utx-1. Cell reports 23, 2392-2404 (2018).

15. Klosin A, Casas E, Hidalgo-Carcedo C, Vavouri T, Lehner B. Transgenerational transmission of environmental information in C. elegans. Science 356, 320-323 (2017).

16. Moore RS, Kaletsky R, Murphy CT. Piwi/PRG-1 Argonaute and TGF- $\beta$ Mediate Transgenerational Learned Pathogenic Avoidance. Cell, (2019).

17. Kaletsky R, Moore RS, Vrla GD, Parsons LR, Gitai Z, Murphy CT. C. elegans interprets bacterial noncoding RNAs to learn pathogenic avoidance. Nature 586, 445-451 (2020).

18. Alonso-Magdalena P, Rivera FJ, Guerrero-Bosagna C. Bisphenol-A and metabolic diseases: epigenetic, developmental and transgenerational basis. Environmental epigenetics 2, dvw022 (2016).

19. Wan Q-L, et al. N6-methyldeoxyadenine and histone methylation mediate trans-generational survival advantages induced by hormetic heat stress. Science Advances, (2020) (In Press).

20. Li Z, Yu Z, Cui C, Ai F, Yin D. Multi-generational obesogenic effects of sulfomethoxazole on Caenorhabditis elegans through epigenetic regulation. J Hazard Mater 382, 121061 (2020).

21. Nomura T, Horikawa M, Shimamura S, Hashimoto T, Sakamoto K. Fat accumulation in Caenorhabditis elegans is mediated by SREBP homolog SBP-1. Genes \& nutrition 5, 17-27 (2010).

22. Magner DB, et al. The NHR-8 nuclear receptor regulates cholesterol and bile acid homeostasis in C. elegans. Cell metabolism 18, 212-224 (2013). 
23. Pathare PP, Lin A, Bornfeldt KE, Taubert S, Van Gilst MR. Coordinate regulation of lipid metabolism by novel nuclear receptor partnerships. PLoS genetics 8, e1002645 (2012).

24. Goh GY, et al. NHR-49/HNF 4 integrates regulation of fatty acid metabolism with a protective transcriptional response to oxidative stress and fasting. Aging cell 17, e12743 (2018).

25. Watts JL. Fat synthesis and adiposity regulation in Caenorhabditis elegans. Trends in endocrinology and metabolism: TEM 20, 58-65 (2009).

26. Goudeau J, Bellemin S, Toselli-Mollereau E, Shamalnasab M, Chen Y, Aguilaniu H. Fatty acid desaturation links germ cell loss to longevity through NHR-80/HNF4 in C. elegans. PLoS biology 9 , e1000599 (2011).

27. Ogg S, et al. The Fork head transcription factor DAF-16 transduces insulin-like metabolic and longevity signals in C. elegans. Nature 389, 994-999 (1997).

28. Lee SS, Kennedy S, Tolonen AC, Ruvkun G. DAF-16 target genes that control C. elegans life-span and metabolism. Science 300, 644-647 (2003).

29. Brock TJ, Browse J, Watts JL. Genetic regulation of unsaturated fatty acid composition in C. elegans. PLoS genetics 2, e108 (2006).

30. Pinkston-Gosse J, Kenyon C. DAF-16/FOXO targets genes that regulate tumor growth in Caenorhabditis elegans. Nature genetics 39, 1403-1409 (2007).

31. Sen P, Shah PP, Nativio R, Berger SL. Epigenetic Mechanisms of Longevity and Aging. Cell 166, 822839 (2016).

32. Han S, Schroeder EA, Silva-Garcia CG, Hebestreit K, Mair WB, Brunet A. Mono-unsaturated fatty acids link H3K4me3 modifiers to C. elegans lifespan. Nature 544, 185-190 (2017).

33. Merkwirth C, et al. Two Conserved Histone Demethylases Regulate Mitochondrial Stress-Induced Longevity. Cell 165, 1209-1223 (2016).

34. Nikooyeh B, et al. Prevalence of obesity and overweight and its associated factors in urban adults from West Azerbaijan, Iran: the National Food and Nutritional Surveillance Program (NFNSP). Nutrition and Food Sciences Research 3, 21-26 (2016).

35. Brenner S. The genetics of Caenorhabditis elegans. Genetics 77, 71-94 (1974).

36. Wan QL, et al. Intermediate metabolites of the pyrimidine metabolism pathway extend the lifespan of C. elegans through regulating reproductive signals. Aging 11, 3993-4010 (2019).

37. Buis A, et al. Coelomocytes Regulate Starvation-Induced Fat Catabolism and Lifespan Extension through the Lipase LIPL-5 in Caenorhabditis elegans. Cell reports 28, 1041-1049 e1044 (2019).

38. Xiao Y, et al. Caenorhabditis elegans chromatin-associated proteins SET-2 and ASH-2 are differentially required for histone H3 Lys 4 methylation in embryos and adult germ cells. Proceedings of the National Academy of Sciences of the United States of America 108, 8305-8310 (2011).

\section{Figures}


a

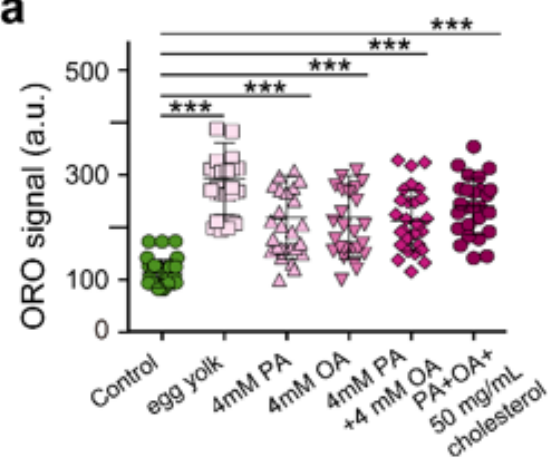

b

은

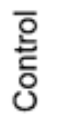

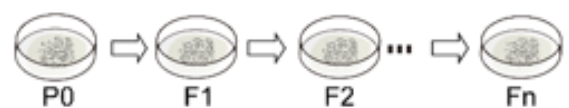

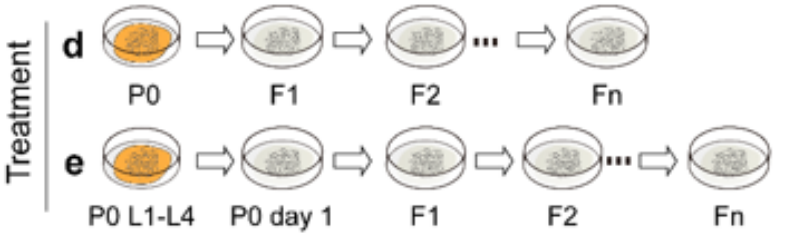

Treatment

C
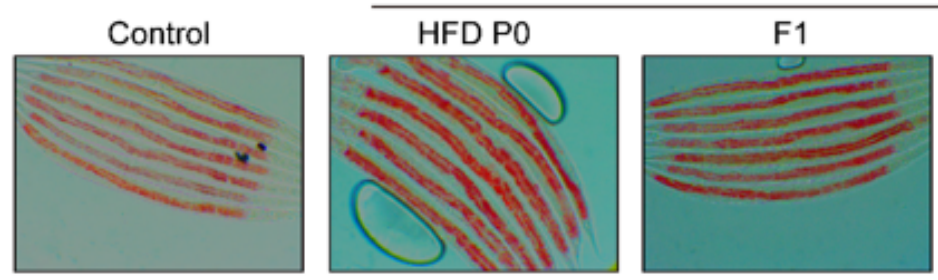

F2

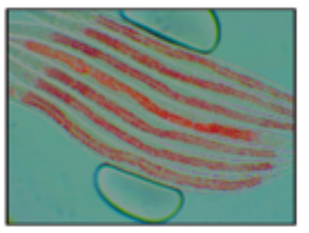

Control Treatment

d

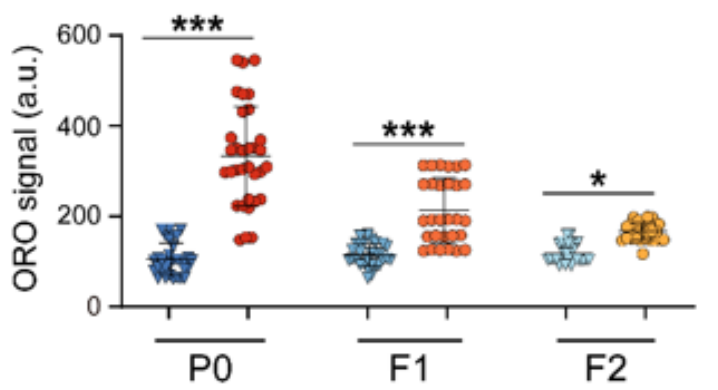

e

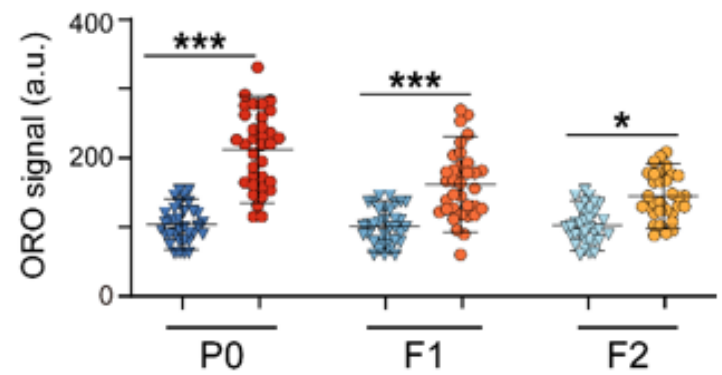

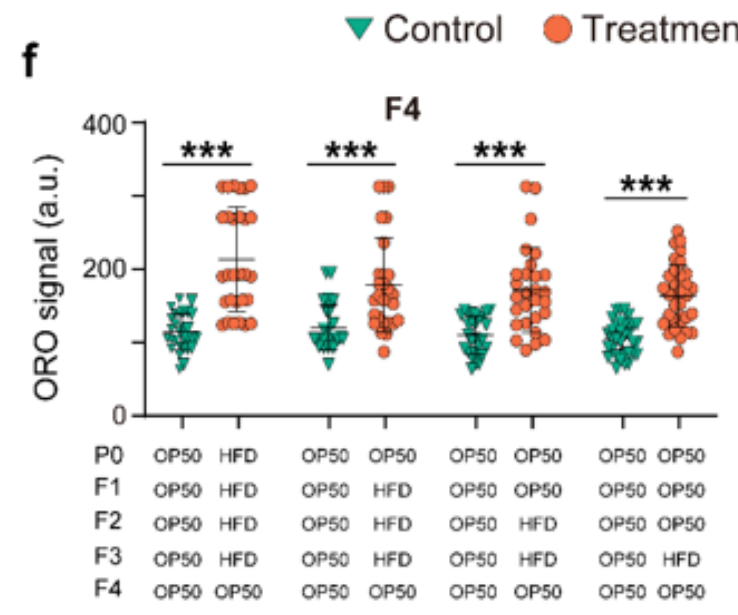

g

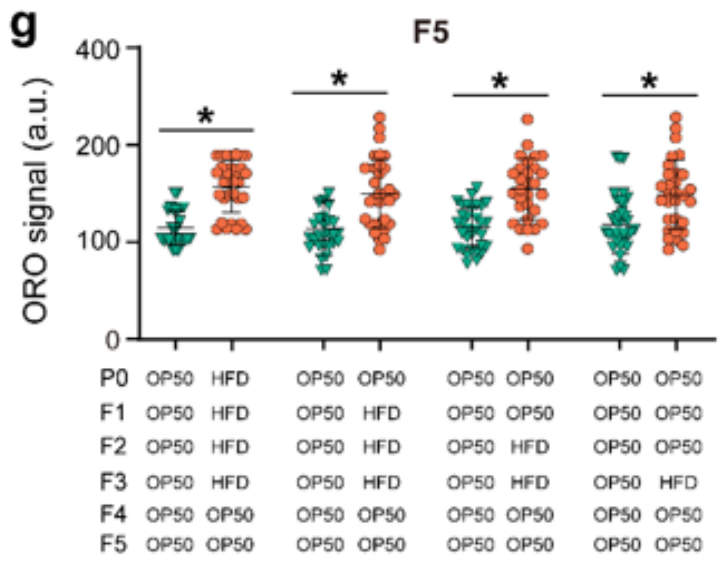

\section{Figure 1}

HFD-induced transgenerational lipid accumulation. (a) Quantification of ORO staining of wild-type (N2) treated with different lipid foods. PA: palmitoleic acid; OA: oleic acid. (b) Experimental scheme. (c, d) Transgenerational inheritance of lipid accumulation induced by HFD (egg yolk) in wild-type C. elegans. (e) Transgenerational inheritance of lipid accumulation induced by HFD in wild-type C. elegans. Here, worms were fed with HFD from L1 to L4 larvae, then they were transferred to normal food until day 1 adulthood. 
Embryos collected by bleaching to obtain F1 animal, and F1 animals were fed with OP50. F2-Fn generations were obtained using the same protocol. ( $f, g)$ Quantification of ORO staining of F1 or F2 wildtype (N2) from parent exposed to HFD four (P0, F1, F2 and F3), three (F1, F2 and F3), two (F2 and F3) and one (F3) generation of animals. Graph data are presented as mean $\pm S D$, statistical analyses were performed by unpaired two-tailed Student's t-test; ${ }^{* \star *} P<0.001,{ }^{* *} P<0.01$, and $* P<0.05$, and the ORO staining values of the replicated tests are listed in Supporting File Table 1.
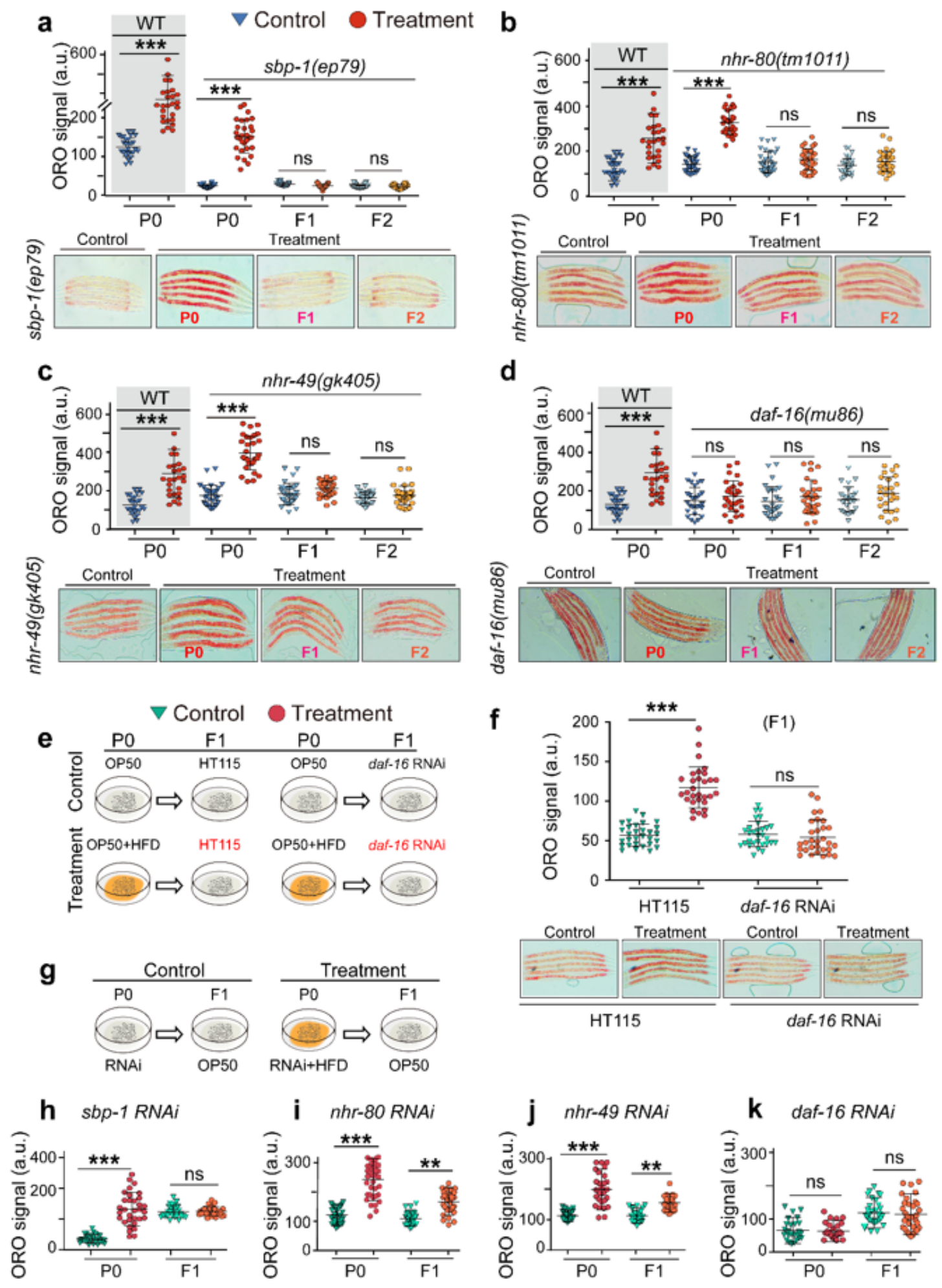

Figure 2 
NHR-49, NHR-80, SBP-1, and DAF-16 contribute to transgenerational inheritance of lipid accumulation. Transgenerational inheritance test of lipid accumulation induced by HFD in sbp-1 mutant (a), nhr-80 mutant (b), nhr-49 mutant (c), and daf-16 mutant (d) (mean \pm SD; Student's t test; ${ }^{\star * * P<0.001, ~}{ }^{\star * P}<0.01$ and ${ }^{*} \mathrm{P}<0.05$, ns: not significant). (e, f) P0 worms were fed with or without the HFD and without any RNAi exposure, and then F1 progeny were exposed to RNAi with daf-16 to test the requirement of daf-16 in F1s. Quantification of ORO staining of F1 worms (mean \pm SD; Student's $t$ test; ${ }^{* \star *} P<0.001,{ }^{*} \mathrm{P}<0.01$, and $\left.{ }^{*} \mathrm{P}<0.05\right)$. ( $\left.\mathrm{g}-\mathrm{k}\right)$ DAF-16 and SBP-1, but not NHR-49 or NHR-80, contributes to transmit the transgenerational information of lipid accumulation. (g) Experimental scheme. (h-k) P0 worms were fed with or without the HFD, and subjected to sbp-1 RNAi (h), nhr-80 RNAi (i), nhr-49 RNAi (j) and daf-16 RNAi (k), then F1 generation raised at OP50. Quantification of ORO staining of F1 worms (mean \pm SD; Student's $t$ test; $* \star * P<0.001, * * P<0.01$, and $* P<0.05$ ). 
a

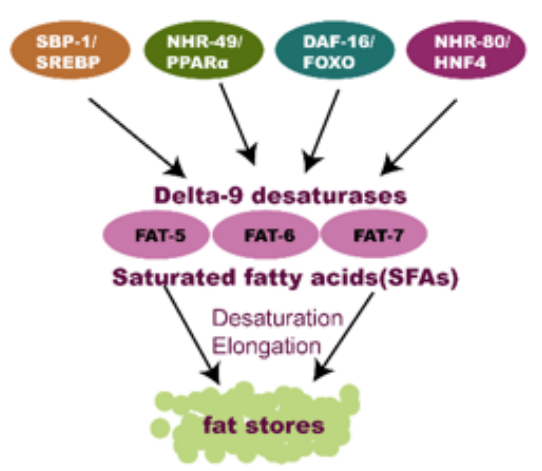

C

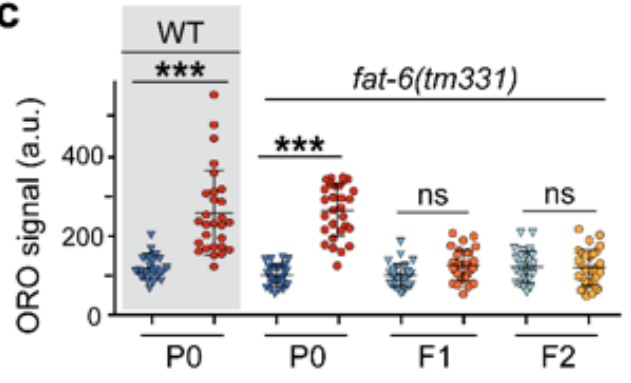

e

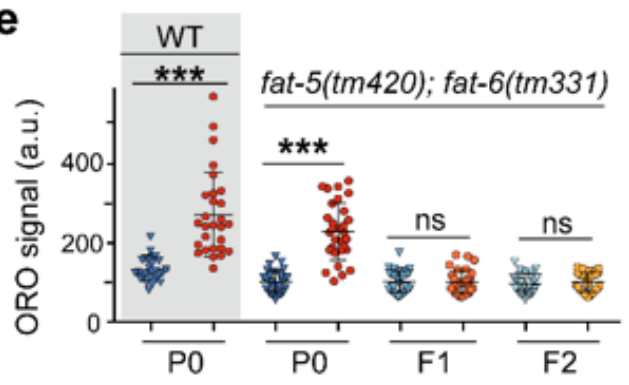

g

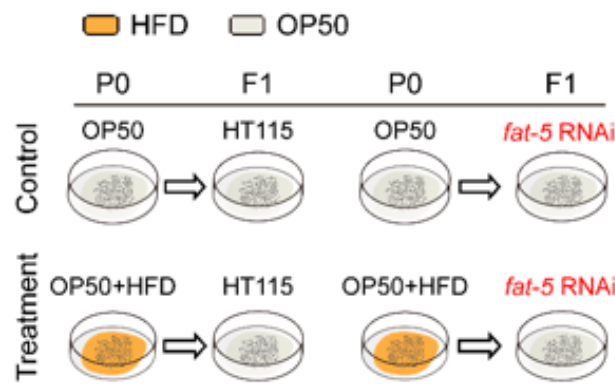

h

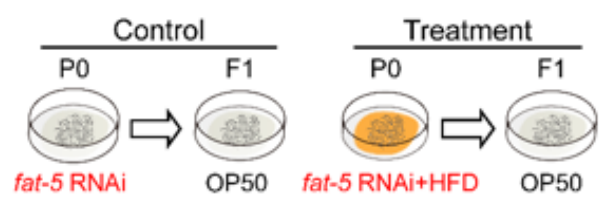

b $\quad \nabla$ Control Treatment

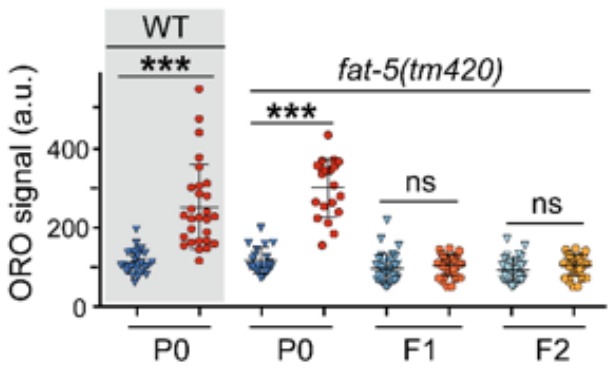

d

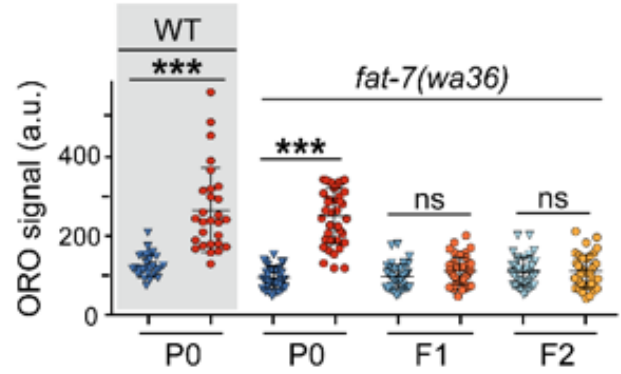

f
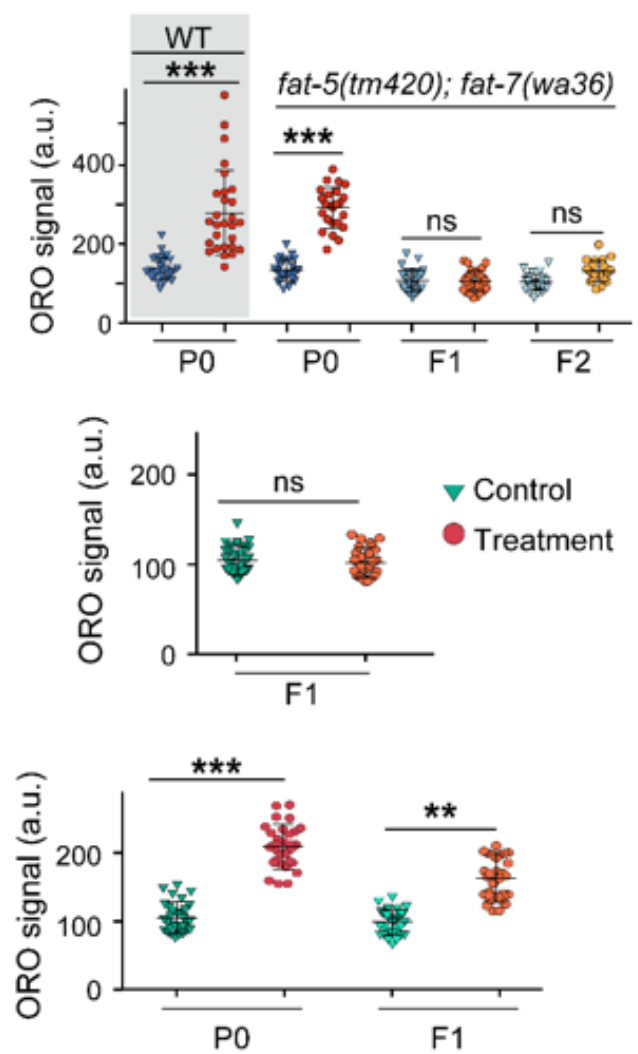

\section{Figure 3}

Transgenerational inheritance of lipid accumulation depends on fat-5, fat-6, fat-7. (a) Transcriptional network regulating delta-9 desaturase gene, modified from Han S, et al. Nature, 2017, 544(7649): 185. Transgenerational inheritance test of lipid accumulation induced by the HFD in fat- 5 mutant (b), fat- 6 mutant (c), fat-7 mutant (d), fat-5; fat-6 double mutant (e), and fat-5; fat-7 double mutant (f) (mean \pm SD; Student's t test; ${ }^{* *} P<0.001,{ }^{*} \mathrm{P}<0.01$ and $\left.{ }^{*} \mathrm{P}<0.05\right)$. (g) P0 worms were fed with or without HFD, and F1 
progeny were exposed to fat- 5 RNAi to determine the requirement of fat-5 in the F1 generation. (h) P0 animals were fed with or without HFD and subjected to fat-5 RNAi, and then lipid level of F1 progeny were analyzed. Graph data are presented as mean $\pm S D$, statistical analyses were performed by unpaired twotailed Student's t-test; ${ }^{* * *} P<0.001,{ }^{*} \mathrm{P}<0.01$, and ${ }^{*} \mathrm{P}<0.05$, and the $\mathrm{ORO}$ staining values of the replicated tests are listed in Supporting File Table 1.

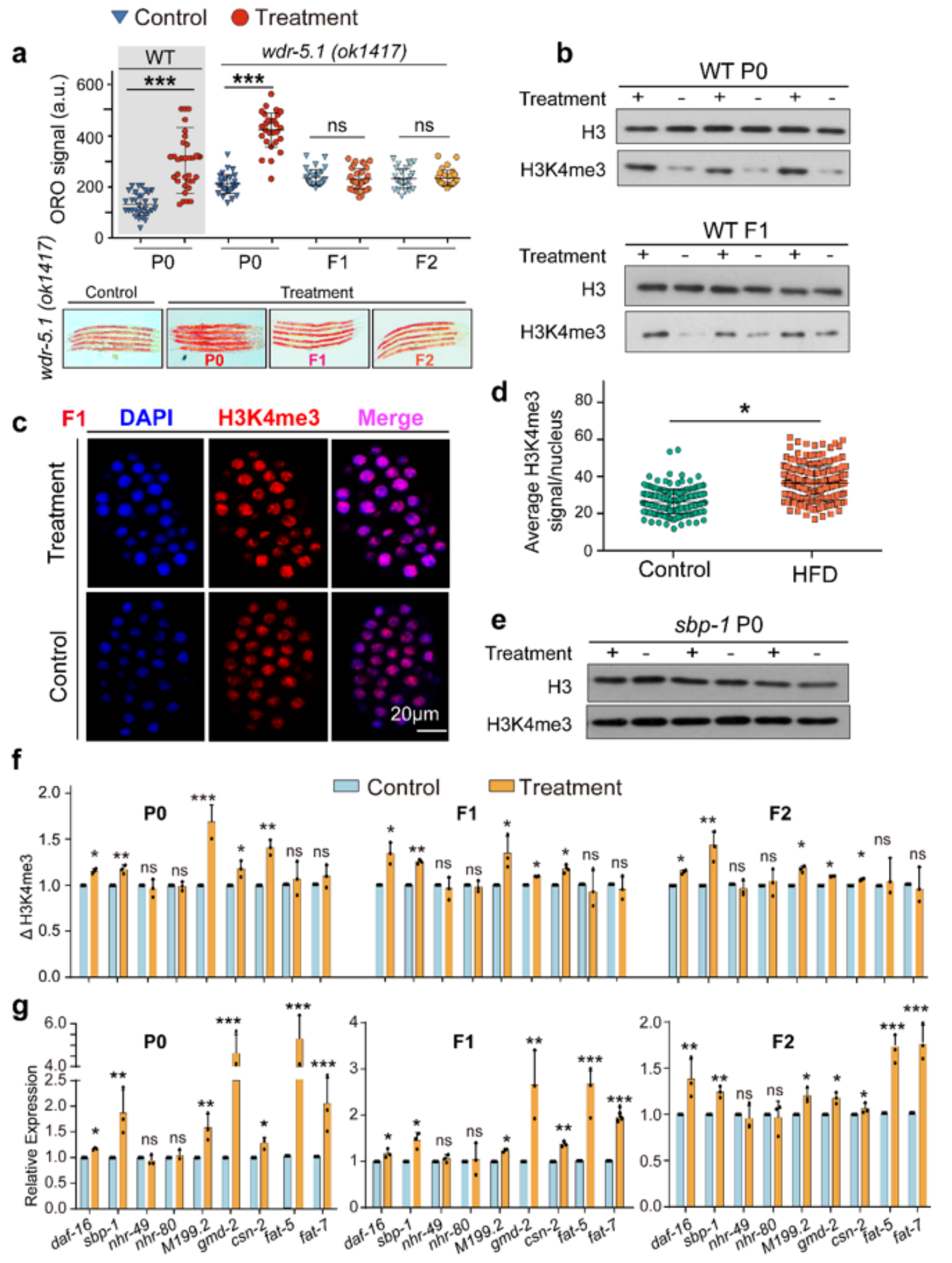

Figure 4 
Histone H3K4me3 modification mediates the transgenerational inheritance of lipid accumulation. (a) Transgenerational inheritance test in wdr-5.1 mutant (mean \pm SD; Student's $t$ test; ${ }^{\star * * P<0.001, ~}{ }^{* * P} \mathrm{P}<0.01$ and ${ }^{*} \mathrm{P}<0.05$ ). (b) The level of histone H3K4me3 modification in PO animals fed with or without the HFD, and their recovered progeny. Immunostaining image (c) and quantification (d) analyzed the level of histone H3K4me3 modification in F1 embryos from parents fed with or without the HFD. Nuclei in blue, H3K4me3 in red. Mean \pm SD from 3 independent experiments, each with $n=10-20$ nuclei per worms, $n \geq$ 20 worms per condition. (e) The level of histone H3K4me3 modification in sbp-1 mutant fed with or without the HFD. (f) H3K4me3 CHIP-qPCR of lipid accumulation response genes of wild-type animals fed with or without the HFD and their recovery F1 and F2 progeny. (g) The mRNA level of genes detected in the (f). Mean \pm SD; Student's t test; ${ }^{* \star *} P<0.001,{ }^{\star *} P<0.01$ and ${ }^{*} P<0.05$, ns: no significance. 
a

Germline and intestine specific RNAi AMJ345

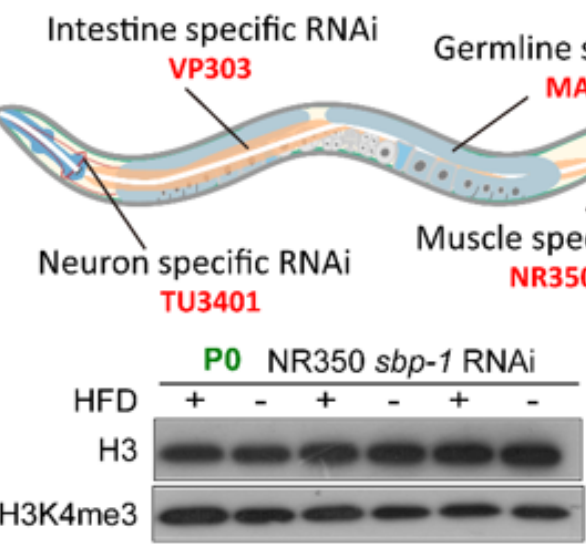

e

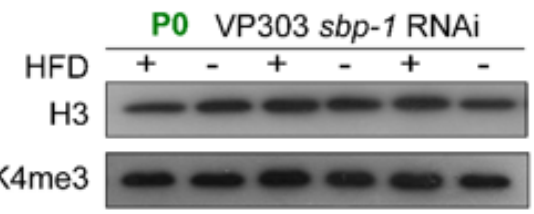

b

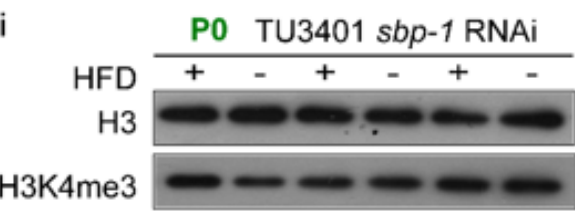

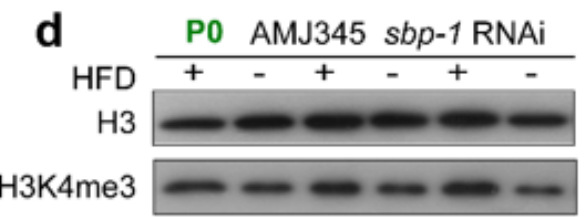

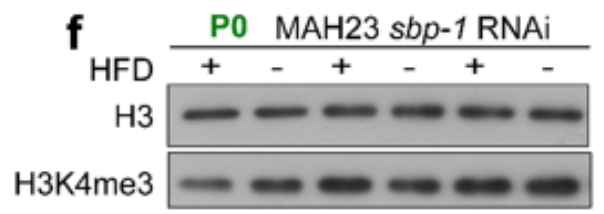

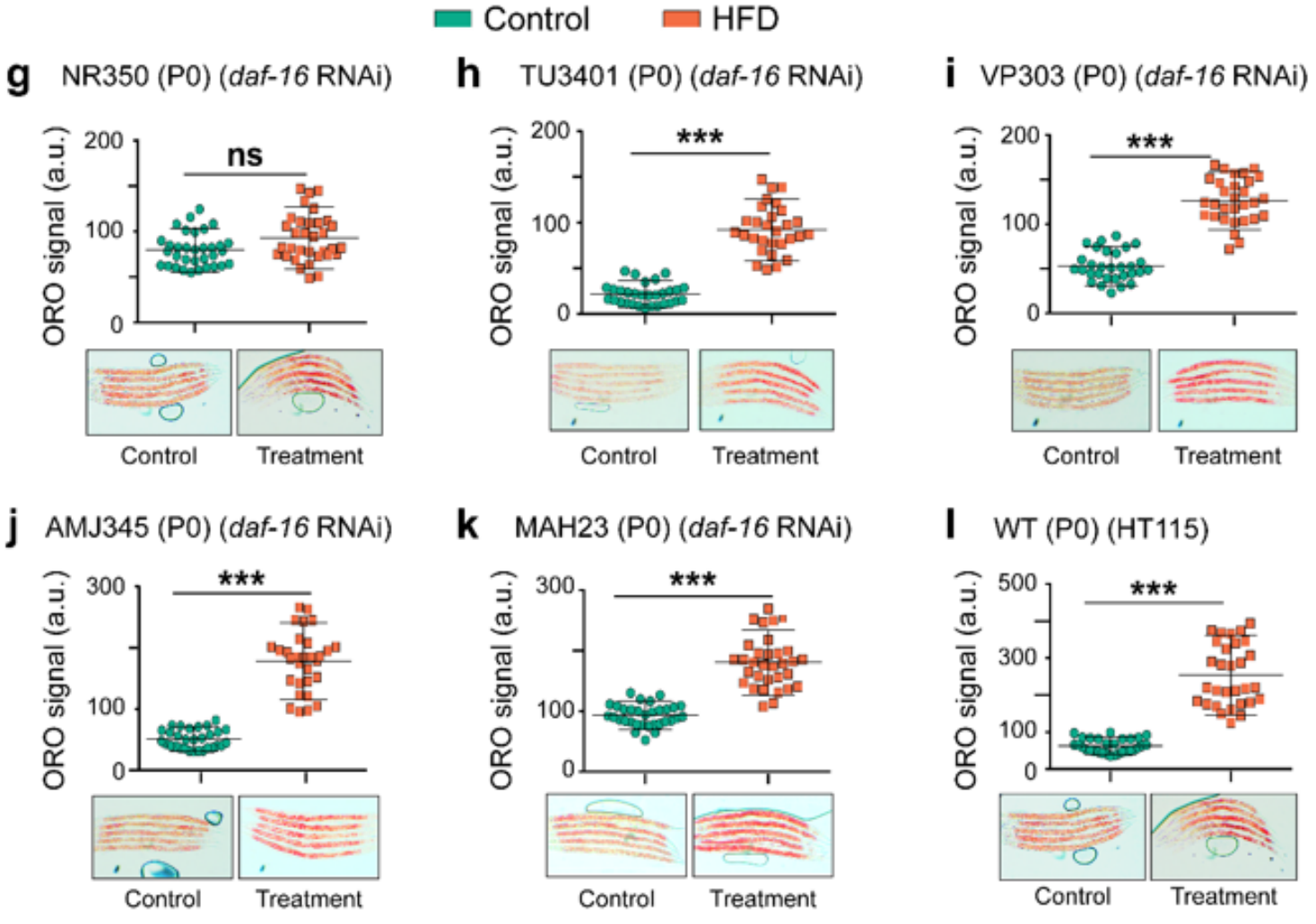

\section{Figure 5}

Tissue-specific RNAi of daf-16 or sbp-1. (a) Strains used for the tissue-specific gene knockdown. (b-f) The level of histone H3K4me3 modification in worms subjected to neuron-specific sbp-1 RNAi (b), musclespecific sbp-1 RNAi (c), germline and intestine-specific sbp-1 RNAi (d), intestine-specific sbp-1 RNAi (e), germline-specific sbp-1 RNAi (f). (g-l) quantification of ORO staining in animals subjected to musclespecific daf-16 RNAi (g), neuron-specific daf-16 RNAi (h), intestine-specific daf-16 RNAi (i), germline and 
intestine-specific daf-16 RNAi (j), germline specific daf-16 RNAi (k) and without any RNAi exposure (l). Mean \pm SD; Student's t test; ${ }^{\star \star *} P<0.001,{ }^{*} \mathrm{P}<0.01$ and ${ }^{*} \mathrm{P}<0.05$, ns: no significance.

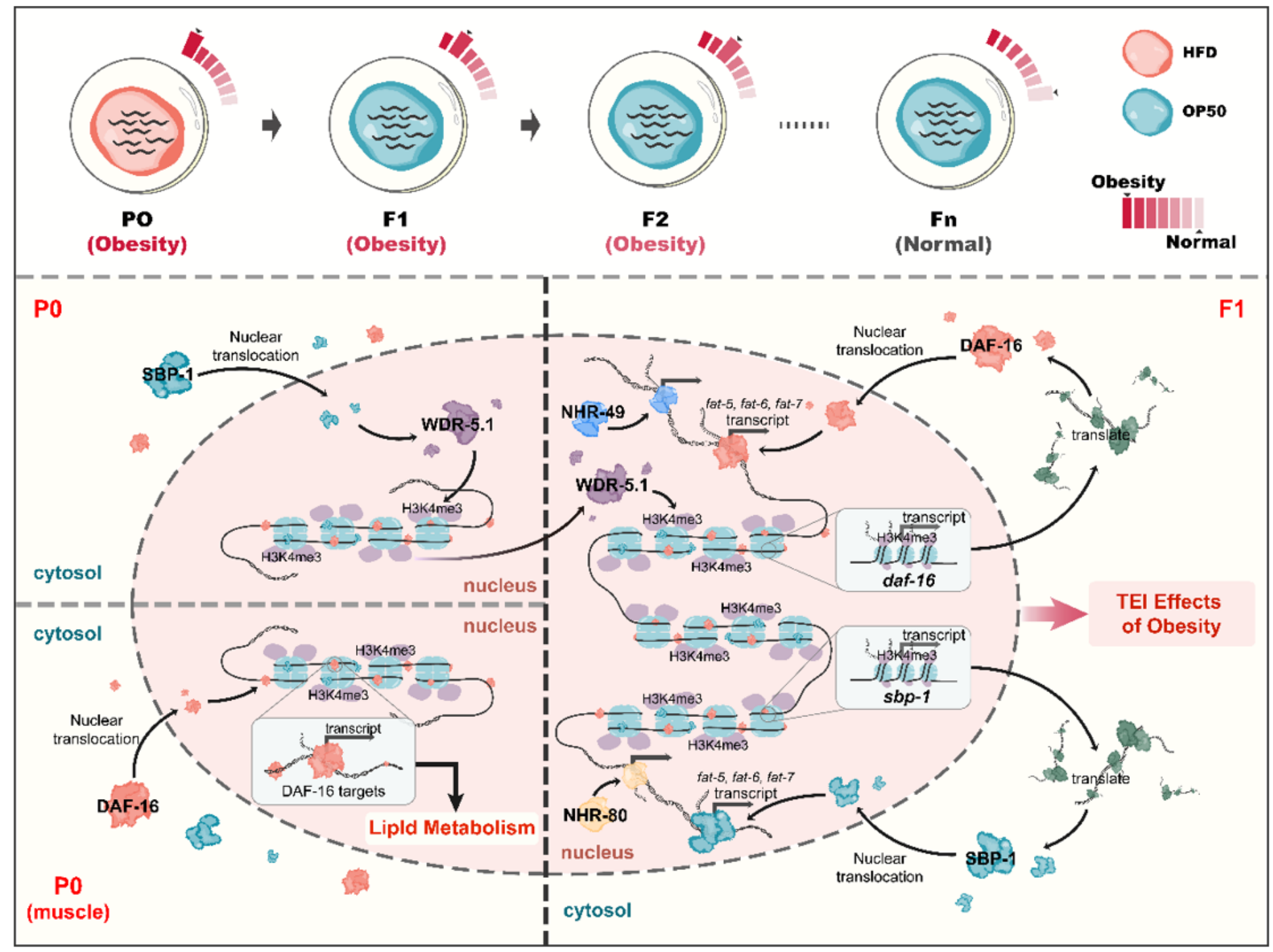

Figure 6

Overview model of transgeneration inheritance in response to lipid accumulation induced by the HFD

\section{Supplementary Files}

This is a list of supplementary files associated with this preprint. Click to download.

- nrreportingsummarylipidTEI002.pdf

- supportinginformation.docx 